\title{
A Directional Change Based Trading Strategy with Dynamic Thresholds
}

\author{
Nora Alkhamees \\ School of Computer Science and Electrical Engineering \\ University of Essex \\ Colchester, UK \\ Email: nyaalk@essex.ac.uk
}

\author{
Maria Fasli \\ School of Computer Science and Electrical Engineering \\ University of Essex \\ Colchester, UK \\ Email:mfasli@essex.ac.uk
}

\begin{abstract}
Traders always seek a trading strategy that can increase and maximize their profits. However, given the current challenges in financial time-series streams - data elements (tick prices) arrive in real-time or almost real-time and at high velocity (at finer time scales) - it is difficult to identify and spot the best time and the most profitable price for trading. The Directional Change (DC) is an event-based approach for summarizing price movements based on a fixed given threshold value. An event in the DC approach is detected if the price change between two points satisfies the given threshold value. In this research, we aim to present a trading strategy based on the DC approach and a dynamic threshold to replace the fixed given one. We call this strategy, the Dynamic Threshold Trading Strategy (DT-TS). Thus, once a DC event is detected (a price change is identified) using the defined dynamic threshold, a trading action is triggered as prices continue to increase or decrease depending on the detected DC event. The trading action to be taken (buy or sell) depends on the previous day price transitions. An experiment was conducted on the FTSE 100 minute-by-minute prices stream to evaluate the DT-TS against different fixed threshold values and different trading strategies. Results showed that the DT-TS was the most profitable strategy among different fixed thresholds and all other examined trading strategies.
\end{abstract}

\section{Introduction}

Identifying significant price movements is crucial in Financial Markets (FMs) as they represent investment opportunities. Traditional methods for observing price fluctuations in financial time-series are based on physical time changes, which depends on a fixed time period of the trading session (e.g., daily closing price), however this fails to capture the full activity of price movements [1].

Using this scale (the physical time intervals) nowadays, it is too difficult and challenging to observe and track price movements in financial time-series streams (intraday prices), as this depends on a fixed time period rather than having a more flexible approach to looking at time intervals. The Directional Change (DC) approach in contrast, observes price fluctuations depending on intrinsic time rather than physical time [1]. Intrinsic time is an event based timing system (irregularly spaced in time), as opposed to the physical time, which is a point based timing system depending on fixed time intervals [2]. The DC [3] is an event-driven approach for summarizing price movements consisting of two type of events, a downturn and an upturn event. A DC event is detected if the market price change satisfies a fixed given threshold value.

Generally in FMs, automated trading strategies embed technology when trading. Thus, computers can automatically trade after defining a set of rules by setting the price, type of trade (buy or sell) and the quantity to invest [4]. Artificial intelligence, Data mining and Machine learning can be used to generate trading rules depending on historical market data. However, it is hard to review the entire market history, with streams of financial data arriving in almost real-time and at high velocity, especially when they are essentially streams of unbounded size. As a result, in this work we aim to use the DC approach and the previous day price change (short history) to define our trading rules.

A successful trade is one which takes place at the right time, with a good final price. The determination of the most profitable threshold value has always been an issue in the DC approach when applied with trading strategies. Different studies have considered different ways for specifying the best threshold value as in [5], [6]. However, in this research, we present a trading strategy that is based on the DC approach along with using a daily dynamic calculated threshold based on what has happened in the previous day to replace the fixed given one. We consider a DC event as an opportunity for trading as a price change was identified. Thus, once a DC event is detected and a price increase/decrease is noticed, then a trading action is activated. The trading decision, either buying or selling, depends on the previous day price transitions.

The rest of this paper is organized as follows. Next a brief description of the related work is presented. Section 3 provides the DC dynamic threshold definition. The following section discusses our DC based trading strategy, which is the core of this paper. The subsequent section (Section 5) presents the experimental work, where we present, discuss and analyse both the experiments conducted and the findings. The paper ends with a summary and the conclusions. 


\section{Related Work}

\subsection{Directional Change Approach}

The Directional Change (DC) [3] is an event based framework used in financial markets to summarize price movements. A DC event is triggered if the price change satisfies a fixed given threshold value. An event in the DC approach can be either a Downturn Event, or an Upturn Event. The period between a Downturn Event and the next Upturn Event is named a Downward Run, while an Upward Run is the period between an Upturn Event and the next Downturn Event.

In a Downward run, the Last Low price $\left(p_{l}\right)$ is continually updated to either the current market price $p(t)$ or the last low price, whichever is lower. Similarly, in an Upward run, the Last High price $\left(p_{h}\right)$ is continually updated to either the current market price $p(t)$ or the last high price, whichever is higher. Initially the Last Low price $\left(p_{l}\right)$ and Last High price $\left(p_{h}\right)$ are set to $p\left(t_{0}\right)$ the initial market price at time $t_{0}$.

An Upturn Event is identified during a Downward run when the current price $p(t)$ exceeds the last low price $\left(p_{l}\right)$ by a given fixed threshold $\Delta x_{d c}$, see equation 1 .

$$
p(t) \geq p_{l} *\left(1+\Delta x_{d c}\right)
$$

While a Downturn Event is identified during an Upward run when the current price $p(t)$ is lower than the last high price $\left(p_{h}\right)$ by a given fixed threshold $\Delta x_{d c}$, see equation 2 .

$$
p(t) \leq p_{h} *\left(1-\Delta x_{d c}\right)
$$

A Downturn Event starting point is called a Downturn Point (the point where the price last peaked), while the end point is called a Downturn Confirmation Point (the point where the price fell by the fixed threshold from the Downturn Point). Similarly, an Upturn Event starts by a point called an Upturn Point (the point where the price last dropped down), while the end point is called an Upturn Confirmation Point (the point where the price rose by the fixed threshold from the Upturn Point).

An Overshoot (OS) event occurs at the end of the current DC event (either an upturn or a downturn event) and lasts until the beginning of the next DC event. An OS event can be either a Downward Overshoot Event, which follows a Downturn Event, or an Upward Overshoot Event, which follows an Upturn Event. Together an Upturn event and an Upward OS form an Upward Run, and a Downward Run on the other hand is formed of a Downturn event and a Downward OS. Figure 1 shows the DC concepts demonstrated in a graph using our FTSE 100 data stream. It shows a downturn event, which started on 1st Feb at 8:01 am (downturn point) and ended on 2nd Feb at 8:36 am (downturn confirmation point), a downward OS event started on 2nd Feb at 8:37 am and ended on 11th Feb 9:30 (the beginning of the next upturn event). The algorithm for the detection of DC events can be found in [7].

The DC approach has shown its potential for studying and analysing financial time-series for the Foreign Exchange
Market [1], [3], [7], [8], [9], [10], [11], [12], [13], and has the potential to be applied to other market data [14], [15], [16], [17]. Furthermore, the DC approach has been part of works related to trading strategies [5], [6], [13], [17] and forecasting [10], [12], [14], [18] studies. In addition, it has typically been applied with a fixed threshold, whereas a dynamic threshold may be more appropriate for markets whose nature is inherently dynamic.

\subsection{DC based Trading Strategies}

One study that presents a trading strategy based on the DC approach is in [17]. In this work, the authors proposed a trading strategy by combining the DC approach with a Genetic Programming (GP) algorithm. More specifically, the GP algorithm combines the use of different threshold values to present a trading strategy. They found that the combination of multiple thresholds helps to target buy or sell strategies at more favourable times.

Another effort was a trading strategy named the Directional-Change Trading (DCT1) which was proposed in [5]. The main idea was based on a learning process from historical data where the most profitable trading strategy (Trend Following or Contrarian Trading) and threshold value are found before actually trading in the market.

Trend Following (TF) [19] is a rule-based trading technique where buying and selling orders are made according to the market trend, it neither forecasts nor predicts the market movement. The TF requires setting up the trading rules prior to trading, once a trend is identified, the trading rules are activated until the next trend is identified. Basically a TF trader makes a buy order when the prices are rising, and a sell order when the prices are falling. The Contrarian Trading (CT) technique on the other hand acts in the opposite way with regard to the trend direction. More specifically, a CT trader makes a sell order when the prices are rising, and a buy order when the prices are falling.

More recent work in [13] presented a contrarian trading strategy based on the DC concept named the Backlash Agent (BA). In a downward run, if Overshoot Value $(O S V)$ is less than a threshold called (Down-ind), then a buy order is opened. The order is closed when the DC confirmation point of the next upturn event is confirmed. The opposite happens when it is an upward run, if the $O S V$ value exceeds a given threshold (Up-ind) then a sell order is opened, while a buy signal is generated when a new DC event (downturn event) is found. Equation 3 describes how the $O S V$ is calculated, where theta is the static threshold value, and $P_{D C C *}$ is the highest/lowest price during an upward/downward OS event.

$$
O S V=\frac{\left(\frac{\left(P_{t}-P_{D C C *}\right)}{P_{D C C *}}\right)}{\text { theta }}
$$

As it was hard to find the most profitable value for the threshold Down-ind and Up-ind, the authors tried to automatically set it by applying 100 Down-ind/Up-ind values on a training dataset prior to trading. The value with the highest profit was chosen as the most suitable value for the threshold 


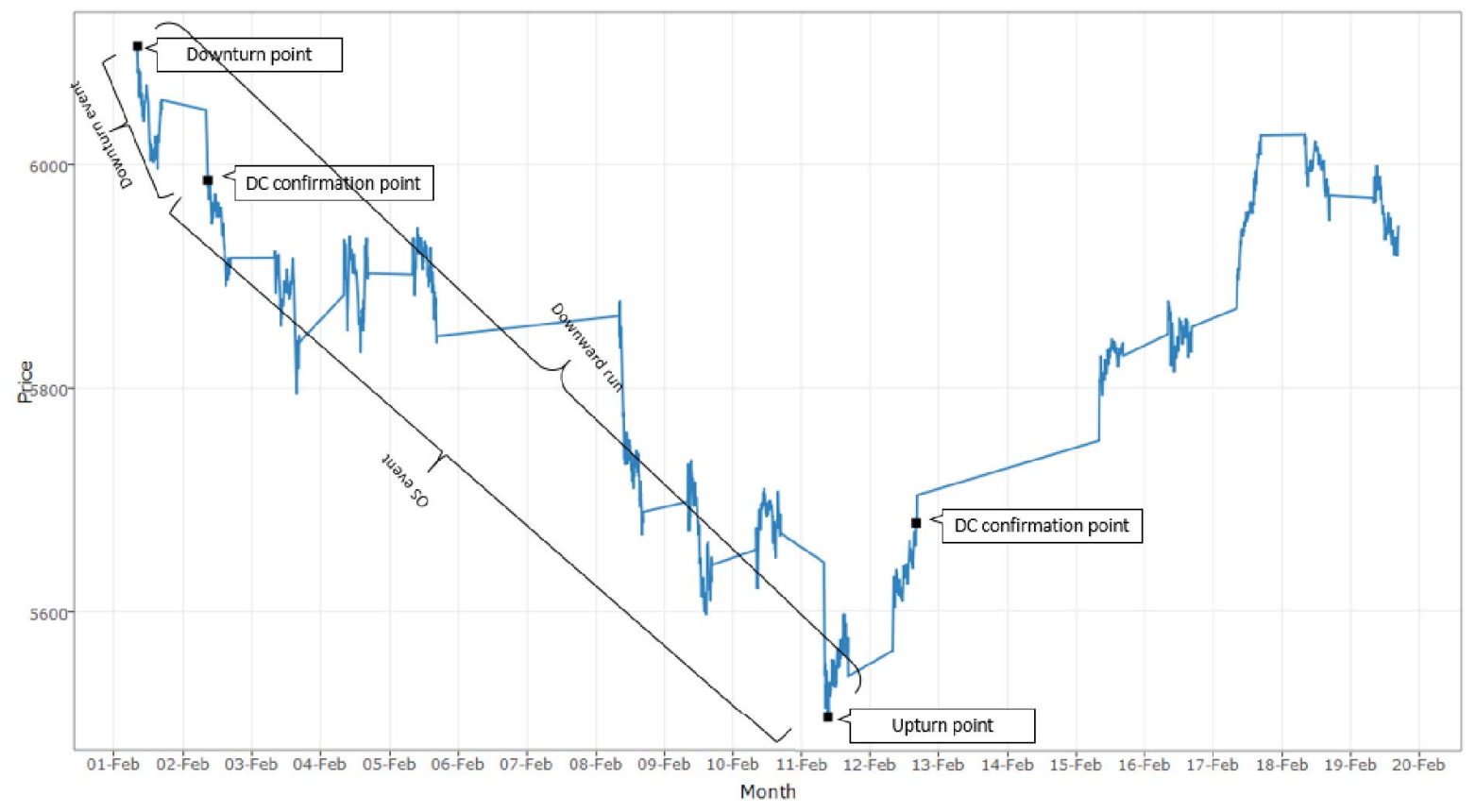

Figure 1. DC Concepts

Down-ind/Up-ind and is used when actually trading in the period under consideration.

\section{The DC Dynamic Threshold Definition}

In DC, an event is detected if the price change exceeds/falls below the given fixed threshold value. However, and depending on the fixed threshold value, a significant event may be missed if the threshold is not satisfied. Consider the following scenario during an upward run and we have a fixed threshold value $1 \%$. Suppose a sudden price decrease by $0.99 \%$ takes place during the current day, but as the threshold is not satisfied no downturn event is detected although the price decrease is significant and it also misses the threshold by a very small value. However, a few days later the prices go down by $0.01 \%$ and in this case a downturn event is detected as the threshold is met. Hence, we suggest that a dynamic threshold that is daily calculated is more appropriate and desirable for event detection, as it can detect events as they occur, so that investors or artificial software agents can detect movements in the market that they can potentially react to and take advantage of. The dynamic threshold is defined based on the price change between the current price $p(t)$ and the last high $\left(p_{h}\right) /$ low $\left(p_{l}\right)$ price, together with what has happened during the previous day. In addition, we have evaluated the detected DC events using the dynamic threshold and different fixed thresholds against what has been released in major news outlets. If a DC event was detected the same day a news event was released then it is said a true event, and a false or spurious event otherwise.
In the following, we describe how the threshold can be calculated on a daily basis. First, we start by calculating the percentage difference of the last high price $p_{h} /$ last low price $p_{l}$ reached during the current upward/downward run and the current day opening price, this captures the basic idea of the DC event detection, refer to equations 4 and 5 for the upward and downward cases.

In the Upward run case:

$$
A=\frac{p_{h}-C p_{\text {open }}}{p_{h}} \times 100
$$

While in the Downward run case:

$$
A=\frac{p_{l}-C p_{\text {open }}}{p_{l}} \times 100
$$

Then we consider what has happened on the previous day, hence we calculate the percentage difference of the previous day opening price $\left(P p_{\text {open }}\right)$ and closing price $\left(P p_{\text {close }}\right)$ to discover price transitions that have happened the previous day. The higher the percentage difference, the higher the likelihood that an event has happened, see equation 6 .

$$
B=\frac{P p_{\text {open }}-P p_{\text {close }}}{P p_{\text {open }}} \times 100
$$

Additionally, we also consider what has happened overnight, thus we calculate the percentage difference of the previous day closing price $\left(P p_{\text {close }}\right)$ and current day opening price $\left(C p_{\text {open }}\right)$ to discover price transitions taking place between the previous day closing price and current day opening price (overnight). Again the higher the percentage, 
the higher the likelihood that an event has happened/is currently unfolding, see equation 7.

$$
C=\frac{P p_{\text {close }}-C p_{\text {open }}}{P p_{\text {close }}} \times 100
$$

Both the previous day and overnight percentage difference are considered as indicators of an event happening if the difference between the opening and closing price is big. The overall threshold is the sum of the aforementioned metrics $\mathrm{A}, \mathrm{B}$, and $\mathrm{C}$ (as per equation 8 ), unless something extreme is noticed the previous day or overnight (between the previous day closing price and the next day opening price). Thus if something has happened the previous day, then the threshold is the sum of A and B metrics only (refer to equation 9), otherwise if it happened overnight/is still unfolding, then the threshold is the sum of $\mathrm{A}$ and $\mathrm{C}$ metrics only (refer to equation 10). We tried to reduce the threshold value (as in equations 9 and 10), if a price change was noticed in the share prices to ensure the detection of an event as we are confident something has happened the previous day or overnight. Furthermore, this ensure that the threshold value is reduced if something extreme happened the previous day and continues to unfold overnight (between previous day closing price and current day opening price) which will result in too high a threshold value which might not be able to detect events.

$$
\begin{gathered}
\text { Threshold }=A+\left(B \times w_{1}\right)+\left(C \times w_{2}\right) \\
\text { Threshold }=A+\left(B \times w_{1}\right) \\
\text { Threshold }=A+\left(C \times w_{2}\right)
\end{gathered}
$$

In order to avoid detecting events based only on the price change between the current day opening price and $p_{h} / p_{l}$ (the A metric), we have considered adjusting the values of B and $\mathrm{C}$ with small weights to increase their values if they are too small and have no effect. Finally, if the values of metrics $\mathrm{B}$ or $\mathrm{C}$ are already big, these weights are of small value and will still be satisfied throughout the day as prices are increasing or decreasing.

We have examined different values of weights and what they may represent, and we concluded that the metric $\mathrm{C}$ can be given a higher weight than $\mathrm{B}$ as we are almost certain that the price change between the previous day closing price and current day opening price will continue to increase/decrease even more throughout the day (as the price change was spotted overnight), thus a higher weight will still be satisfied as prices will most probably continue to increase/decrease. The values of these weights were assigned as follows:

$$
\begin{aligned}
& w_{1}=15 \% \\
& w_{2}=20 \%
\end{aligned}
$$

An experiment was run for 36 weeks using the FTSE 100 minute-by-minute prices stream from the Reuters Thomson One [20] to detect the occurring DC events using our daily calculated dynamic threshold and four different fixed
TABLE 1. Dynamic and FiXed Thresholds Detected DC Events ANALYSIS

\begin{tabular}{|l||l||l||l|}
\hline Threshold Type & Detected DC Events & $\begin{array}{l}\text { True } \\
\text { Events }\end{array}$ & $\begin{array}{l}\text { False } \\
\text { Events }\end{array}$ \\
\hline Dynamic & 22 & 22 & 0 \\
\hline Fixed $(0.03)$ & 22 & 12 & 10 \\
\hline Fixed $(0.04)$ & 14 & 5 & 9 \\
\hline Fixed $(0.05)$ & 12 & 5 & 7 \\
\hline Fixed $(0.06)$ & 10 & 3 & 7 \\
\hline
\end{tabular}

thresholds ranging from considerably small to bigger values $(0.03,0.04,0.05,0.06)$. All detected DC events by the dynamic threshold or a fixed threshold were evaluated against the released news events in BBC News (www.bbc.co.uk) regarding the studied share (the FTSE 100 index).

Using the dynamic threshold we were able to detect 22 events in the period from July 2015 until the end of March 2016. The dynamic threshold values ranged from 0.014 to 0.071 , refer to figure 2 to see the detected DC events, each event is represented by a black dot.

All the detected DC events by the dynamic threshold matched news headlines, which were published on the same day the DC event was found at closing price. While with the examined fixed thresholds only few of the detected DC events matched news events, they were in most cases identified either before or after a news event was released (refer to table 1 to see an analysis of the detected DC events for the different used thresholds). In more detail, the 0.03 fixed threshold detected the same number of events using the dynamic threshold (22 events), but 10 out of those 22 DC events were detected either before or after the news event. The 0.04 fixed threshold on the other hand missed more DC events than the true detected ones (only 5 out of the 14 detected DC events were true events). With the 0.05 fixed threshold more than half of the detected DC events were missed ( 7 out of 12 DC events). Finally the 0.06 fixed threshold missed $70 \%$ out of the detected DC events (7 out of 10 DC events).

As noticed from using fixed threshold values, events were missed or inaccurately identified, perhaps at the wrong time. This in essence means that fixed thresholds could miss trading opportunities, and therefore this would have an impact on the profit generated by a strategy using fixed thresholds in general.

\section{Our DC based Trading Strategy}

We introduce a trading strategy that is based on the DC approach and our dynamic defined threshold along with considering what has happened in the previous day (short term history). We want to take advantage of what has occurred the previous day before trading, thus we consider the previous day price change as a clue for the type of trading to be adopted. For example, when we are experiencing an upward run and the previous day price change (price increase) was big, then it is a selling opportunity rather than a buying, as 


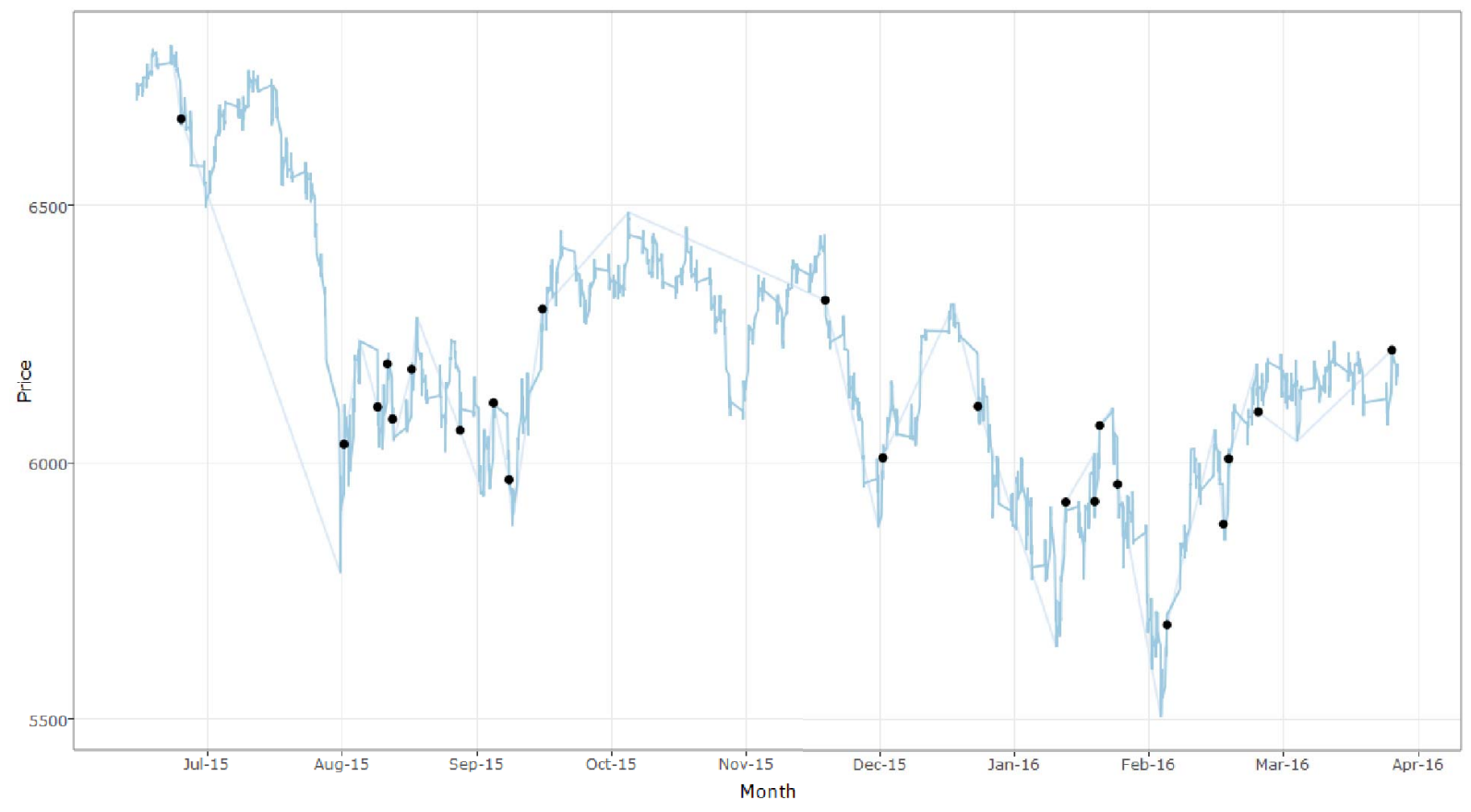

Figure 2. Detected DC Events from July 2015- March 2016

prices increased significantly. Hence we consider a big price change, as a contrarian trading strategy opportunity.

Accordingly if the price change that occurred the previous day was extreme, then we follow a CT strategy (as prices are becoming too high or too low depending on that trend and we do not want to miss that opportunity), and if not, then we follow a TF trading strategy.

Whenever an OS event starts during either a Downward run or an Upward run, we keep track of every price change within that trend (every $p_{h} / p_{l}$ price change). If the change of either $p_{h} / p_{l}$ has been tracking an extreme event during the previous day, then the change of price is supposed to be big, and so we adopt a CT trading strategy. In particular, if the price change was in $p_{l}$ (price decrease), then it is a buying opportunity as prices are becoming too low, otherwise if it is in $p_{h}$ (price increase) then it is a selling opportunity as price are becoming too high. In contrast, if a price change was only identified in $p_{h} / p_{l}$ with nothing special identified during the previous day, then we follow a TF trading strategy. Finally, if the price change was in $p_{l}$ (price decrease), then it is a selling opportunity, otherwise if it is in $p_{h}$ (price increase), then it is a buying opportunity.

In general, if all amount of money and shares are used when trading, a single trading action can take place in every trend (no sequential trading actions i.e. 2 buy or 2 sell trades), as all the amount of money or shares is already used. However, a positive thing about our trading strategy is the ability to continue trading even if the amount of money or shares is already used, as the next trading action might be the opposite way. Hence, if we have already traded and then suddenly the prices sharply decrease or increase, we still can take action and benefit from that price change. This is in contrast to other trading strategies which use the whole amount of money or shares, and they cannot further take advantage of a big price change opportunity, as a trading rule has already been satisfied, and the amount of money or shares is already consumed. In summary, when using our trading strategy, more trading actions can still be executed, even if we are in the same trend and the previous action has used all the amount of money or shares.

An example from our applied experiment was the first detected DC event (a Downward run) starting from 22nd July till 24 August with 4 alternate trading actions taking place (initially the amount of money was $100 \mathrm{k}$ and with zero shares). In particular, the first trading action took place on that downward run and was a sell action on 24th July, the prices were decreasing but nothing extreme was noticed on the previous day or overnight (the share price was 6644). The amount of cash after selling is 104315 (profits were 4315) and shares are zero. As the downward run continued (prices are decreasing), a second trading action was activated when a big price decrease was noticed as prices went sharply down during that day (the share price reached 6585), thus a buying trading action took place (bought shares were 15 and the left cash was 5544). The next trade was a sell action on 19th Aug as nothing extreme was noticed the previous day or overnight (the share price reached 6495), the amount of money after the sell action was 108435 (profits were 8435) and the left shares were zero. Afterwards the prices decreased even more (the share price was 6450 ), so it is a 
buying opportunity (bought shares were 16 and the left cash was 5226).

Alternatively, using the TF trading strategy a single trading action (selling) took place on 22nd July (the share price was 6664) during the downward run (the day the DC event was detected). The amount of cash after sell activity was 104603 (profits 4603) and shares are zero. Using the CT trading strategy a single trading action (buying) took place on 22nd July (the share price was 6664) during the downward run. The bought shares were 15 and left cash was 43. It is clear how our trading strategy performed in comparison to other trading strategies, which also uses the same money management approach (consuming the whole amount of cash or shares when trading).

The previous day price change is said to be extreme if the percentage difference of what has happened the previous day (between previous day opening and closing price) or happened overnight (between previous day closing price and current day opening price) is $0.9 \%$. The percentage was determined as a result of applying the J84 Decision Tree to generate the trading rules for our trading strategy. J48 is the Java open source implementation of C4.5 [21] in WEKA [22]. WEKA is a data mining tool consisting of a collection of machine learning algorithms. We used more than 36 weeks FTSE 100 minute-by-minute prices as our training dataset (from July 2015 till March 2016). The training dataset for each minute shows the following: date and time, share price, percentage difference for previous day opening and closing price (perc1), percentage difference for previous day closing and current day opening price (perc2), whether it is an event confirmation point or not, whether it is an overshoot period and $p_{h} / p_{l}$ has changed, and finally the trading decision. The trading decision can be either Next pt (which means no trading takes place and call the next minute price), or TF, or CT. Figure 3 shows the built decision tree for extracting our trading strategy trading rules from the training dataset. A trading takes place if there is sufficient amount of monetary units or shares and there is a price change during the OS period, if not then the next incoming price is examined. The type of trading to be applied (either CT or TF) depends on the previous day circumstances.

The DT-TS trading rules are as follows:

In a Downward run and during an OS event:

Rule 1: If the percentage difference between the previous day opening and closing price or previous day closing price and current day opening price is greater than or equal $1 \%$, then generate a buy order.

Rule 2: If $p(t)<p_{l}$ (if the current price is less than the lowest reached price) then generate a sell order.

\section{In an Upward run and during an OS event:}

Rule 1: If the percentage difference between the previous day opening and closing price or previous day closing price and current day opening price is greater than or equal $1 \%$, then generate a sell order.

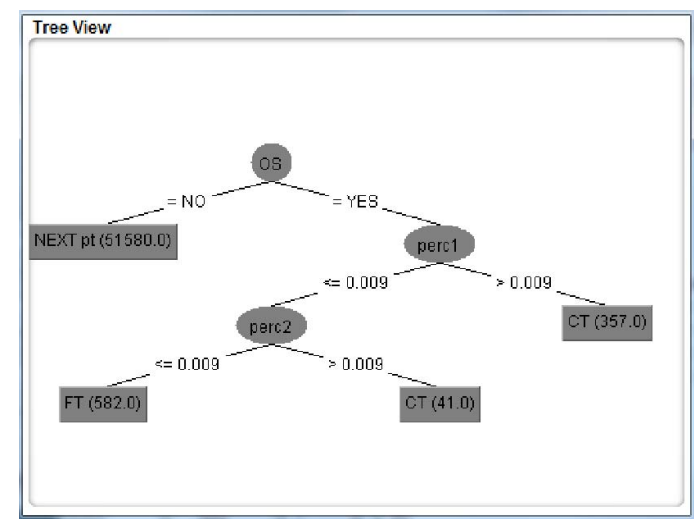

Figure 3. Trading Strategy Decision Tree

Rule 2: If $p(t)>p_{h}$ (if the current price is greater than the highest reached price) then generate a buy order.

In this research and for simplicity, the adopted money management approach is the following: whenever a buy order is activated we use the entire amount of money available, and with sell orders we sell all the available shares. In the applied experiments, we initially start trading with $100 \mathrm{k}$ monetary units and zero shares. We name our trading strategy when combined with our dynamic threshold as Dynamic Threshold-Trading Strategy (DT-TS), and when it is combined with a fixed threshold as Fixed ThresholdTrading Strategy (FT-TS).

\section{Experimental Work}

In this section, we want to demonstrate the applicability of our trading strategy (the DT-TS) when used in conjunction with the DC approach. Furthermore, we want to show that using a dynamic threshold when trading is more profitable than a fixed threshold.

We trained our trading model to generate the trading strategy rules on the FTSE 100 minute-by-minute price stream from the Reuters Thomson One [20]. On a minuteby-minute basis we are obtaining the opening, high, low, and closing price. As there is no big difference in the four prices (opening, high, low, and closing) we are taking the average of them. Figure 2 illustrates the training period and the detected DC events using the DC approach along with our daily dynamic defined threshold. We were able to detect 22 DC events with thresholds of values ranging from (0.0140.071) where each event is represented by a black dot in figure 2 .

In addition, we run the found trading strategy rules using different fixed thresholds (FT-TS), we tried a small fixed threshold (0.03), medium sized thresholds (0.04 and 0.05), and a larger fixed threshold (0.06). Moreover, we tried different trading strategies (CT, TF, and BA) to further compare the profitability and the number of trading actions taking place between our proposed trading strategy and other strategies. Table 2 reveals the profitability of each threshold, 
along with the number of detected DC events, the applied trading strategy, and lastly the number of trading actions taking place. It is clear that the dynamic threshold when used with our trading strategy (DT-TS) outperforms all examined fixed thresholds and all investigated trading strategies (FT$\mathrm{TS}, \mathrm{CT}, \mathrm{TF}$, and BT) with profitability percentage $65 \%$. In general, in the DC approach, low threshold values lead to detecting more events than bigger thresholds, as the former are more easily satisfied than the latter. The more the detected events, the more trading actions may take place, and so the higher the profits reached.

Furthermore, table 2 shows that the same number of detected DC events was found using the dynamic threshold and the 0.03 fixed threshold (22 events), and when using our trading approach the number of trading actions by the 0.03 threshold (FT-TS) were greater than it is by the dynamic threshold (DT-TS), 42 trading actions by the DT-TS in contrast to 45 trading actions by the 0.03 FT-TS. However the dynamic threshold with our trading strategy DT-TS was more profitable (65\%) than the 0.03 fixed threshold FT-TS (62\%) even though the trading actions taking place were less. This is due to events being detected more timely with our dynamic threshold, and so trading actions took place at better points in time and with better prices. Other fixed thresholds $(0.04,0.05$, and 0.06$)$ with the FT-TS in table 2 were less profitable because the number of detected events were less, however the FT-TS still beats the other investigated trading strategies (CT, TF, and BA) when using fixed thresholds. The 0.04 threshold had the highest profitability percentage of 52\% with 14 detected DC events using the FT-TS, while the highest profitability percentage of the 0.05 threshold was $41 \%$ with 12 detected DC events using the FT-TS, and the highest profitability percentage of the 0.06 threshold was $38 \%$ with 10 detected DC events using the FT-TS and the BA trading strategies.

Table 2 demonstrates that the CT trading strategy performs better with high threshold values rather than lower values. While the TF trading strategy on the other hand performs better with low threshold values rather than higher ones. This is because with the TF it is preferable to detect an event as early as possible (with small threshold values), as the trend is expected to continue in the same direction, thus the trading action taken tries to prevent losses when the prices are decreasing and increases the profits when the prices are rising. Using the $\mathrm{CT}$ in contrast, it is preferable to detect the event when the peak has almost been reached (using big threshold values), which means a sell action when prices are rising, and a buy action when prices are dropping. The CT performed best with the 0.06 threshold and the TF performed best with the 0.03 threshold value.

The BA trading strategy [13] was applied on our training set to find the most profitable Down-ind and Up-ind threshold values, which then will be used to trade on the testing dataset.

Using our dynamic threshold it was not possible to find the most profitable value (a single value) of Down-ind and
TABLE 2. Training Period Analysis

\begin{tabular}{|c|c|c|c|c|}
\hline Threshold & $\begin{array}{l}\text { No. } \\
\text { events }\end{array}$ & Trading & $\begin{array}{l}\text { No. } \\
\text { Tradings }\end{array}$ & Profits \\
\hline \multirow{4}{*}{ Dynamic Threshold } & \multirow{4}{*}{22} & DT-TS & 42 & $65 \%$ \\
\hline & & CT & 23 & $34 \%$ \\
\hline & & TF & 23 & $33 \%$ \\
\hline & & $\mathrm{BA}$ & 23 & $38 \%$ \\
\hline \multirow{4}{*}{0.03 (Fixed) } & \multirow{4}{*}{22} & FT-TS & 45 & $62 \%$ \\
\hline & & $\begin{array}{l}\mathrm{CT} \\
\end{array}$ & 22 & $13 \%$ \\
\hline & & TF & 23 & $42 \%$ \\
\hline & & BA & 23 & $37 \%$ \\
\hline \multirow{4}{*}{0.04 (Fixed) } & \multirow{4}{*}{14} & FT-TS & 38 & $52 \%$ \\
\hline & & CT & 14 & $12 \%$ \\
\hline & & TF & 15 & $21 \%$ \\
\hline & & $\mathrm{BA}$ & 12 & $38 \%$ \\
\hline \multirow{4}{*}{0.05 (Fixed) } & \multirow{4}{*}{12} & FT-TS & 30 & $41 \%$ \\
\hline & & CT & 12 & $17 \%$ \\
\hline & & TF & 13 & $7 \%$ \\
\hline & & BA & 12 & $36 \%$ \\
\hline \multirow{4}{*}{0.06 (Fixed) } & \multirow{4}{*}{10} & FT-TS & 23 & $38 \%$ \\
\hline & & CT & 10 & $21 \%$ \\
\hline & & TF & 11 & $-2 \%$ \\
\hline & & BA & 9 & $38 \%$ \\
\hline
\end{tabular}

Up-ind from the training dataset to be used when trading on the testing dataset. This is because we have different threshold value every single day, which results in having a different value for the Down-ind and Up-ind each single day as well. As a result we first calculate the mean of the dynamic threshold values which were of range 0.0140.071 (the dynamic thresholds' mean was 0.0281), then for that mean, we found the value of Down-ind and Up-ind. Accordingly, the value of Down-ind and Up-ind for the mean of the daily calculated threshold values were -0.16 and 0.28 respectively.

Using the 0.03 fixed threshold, the Down-ind and Upind values were -0.05 and 0.01 respectively. While the 0.04 fixed threshold Down-ind and Up-ind values were -0.5 and 0.2 respectively. The 0.05 fixed threshold Down-ind and Upind values were -0.2 and 0.1 respectively. Lastly the 0.06 fixed threshold Down-ind and Up-ind values were -0.6 and 0.12 respectively.

In order to test our trading strategy, we have applied the found trading rules on a testing dataset for the FTSE 100 one minute prices for the period from April 2016 till end of October 2016 with our dynamic threshold DT-TS, and once more, with four different fixed thresholds FT-TS (0.03, 0.04, 0.05, and 0.06). Furthermore we evaluated our trading strategy profitability against the $\mathrm{CT}$, TF, and BA trading strategies. We initially started the trading strategy with 100k monetary units and zero shares. Figure 4 illustrates the detected DC events during the testing period and by using our dynamic threshold, where DC events are shown as small black dots. 


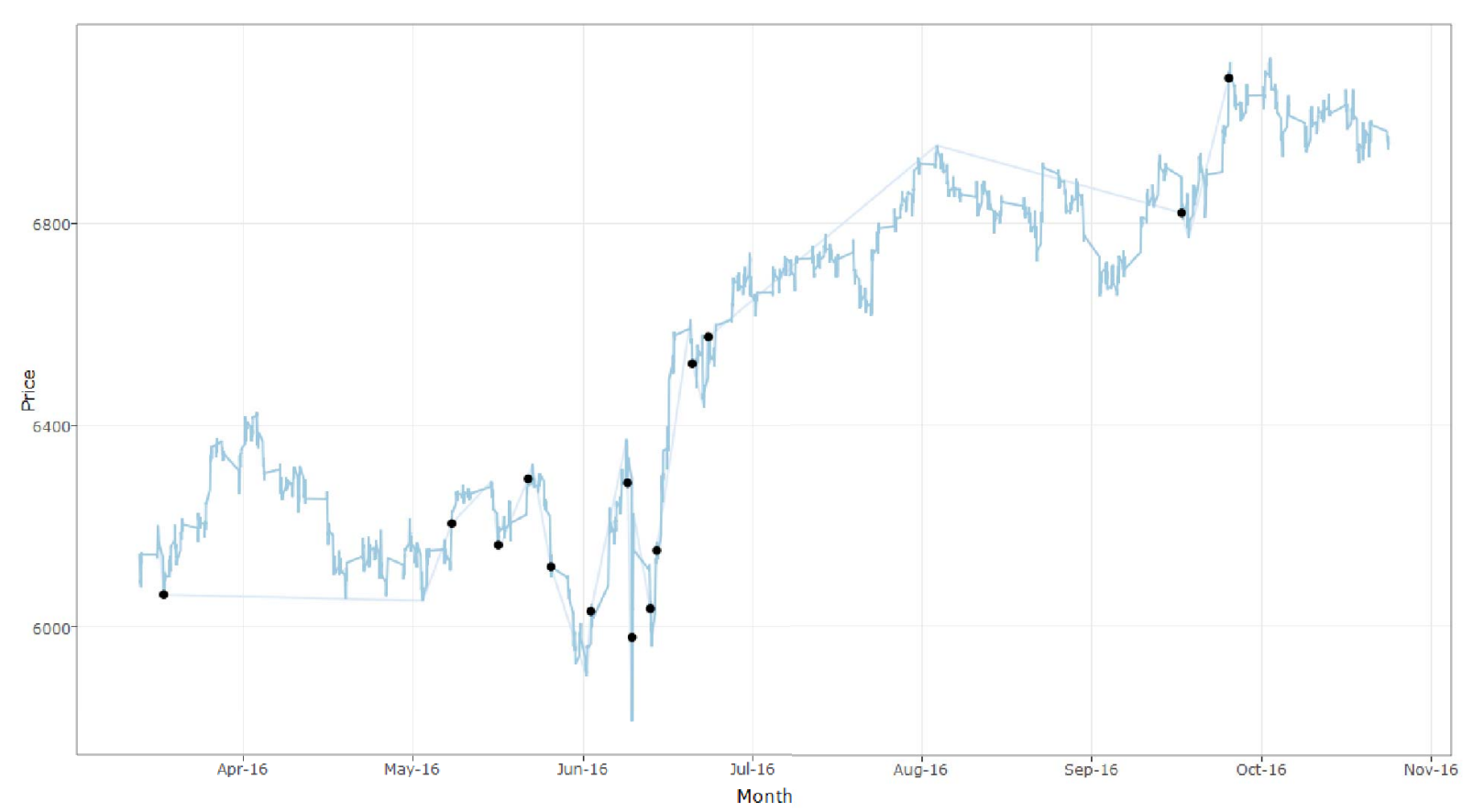

Figure 4. Testing Period and the Detected DC Events

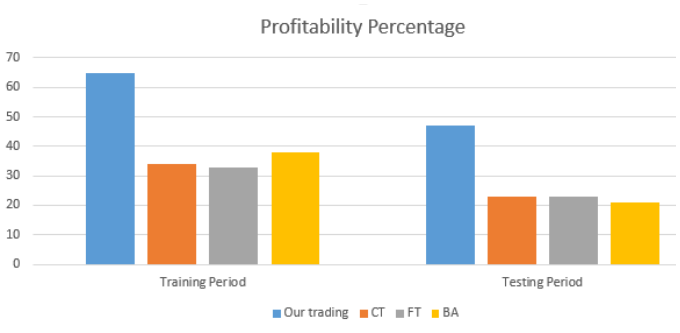

Figure 5. Profitability Comparision between Training and Testing sets

It is clear that the number of the detected DC events in the testing period is small, this is because the market prices were almost going in the same direction (prices were gradually rising), refer to figure 4 . However more than half of the detected events (8 out of 14 events) took place in June 2016, the EU Referendum and Brexit month. This explains why the profitability percentage is generally not high, as Brexit fears were growing. Figure 5 shows a bar chart for comparing between the profitability in the training and testing sets and how our trading strategy when used with a dynamic threshold (DT-TS) outperforms the other investigated trading strategies. In addition, in the testing dataset, our trading strategy with the dynamic threshold (DT-TS) once more outperforms the examined fixed thresholds and the investigated trading strategies (FT-TS, CT, TF, and BA) with $47 \%$ profits, refer to table 3 .

Figure 6 illustrates a bar chart with the profitability in the testing period for all investigated thresholds and trading
TABLE 3. Testing Set AnALysis

\begin{tabular}{|c|c|c|c|c|}
\hline Threshold & $\begin{array}{l}\text { No. } \\
\text { events }\end{array}$ & Trading & $\begin{array}{l}\text { No. } \\
\text { Tradings }\end{array}$ & Profits \\
\hline \multirow{4}{*}{ Dynamic Threshold } & \multirow{4}{*}{14} & DT-TS & 29 & $47 \%$ \\
\hline & & CT & 14 & $23 \%$ \\
\hline & & $\mathrm{TF}$ & 14 & $23 \%$ \\
\hline & & BA & 14 & $21 \%$ \\
\hline \multirow{4}{*}{0.03 (Fixed) } & \multirow{4}{*}{10} & FT-TS & 16 & $35 \%$ \\
\hline & & CT & 10 & $18 \%$ \\
\hline & & $\mathrm{TF}$ & 10 & $20 \%$ \\
\hline & & BA & 10 & $23 \%$ \\
\hline \multirow{4}{*}{0.04 (Fixed) } & \multirow{4}{*}{10} & FT-TS & 15 & $14 \%$ \\
\hline & & CT & 10 & $29 \%$ \\
\hline & & $\mathrm{TF}$ & 10 & $8 \%$ \\
\hline & & $\mathrm{BA}$ & 10 & $17 \%$ \\
\hline \multirow{4}{*}{0.05 (Fixed) } & \multirow{4}{*}{4} & FT-TS & 9 & $17 \%$ \\
\hline & & CT & 4 & $13 \%$ \\
\hline & & $\mathrm{TF}$ & 4 & $10 \%$ \\
\hline & & $\mathrm{BA}$ & 4 & $16 \%$ \\
\hline \multirow{4}{*}{0.06 (Fixed) } & \multirow{4}{*}{4} & FT-TS & 8 & $16 \%$ \\
\hline & & $\mathrm{CT}$ & 4 & $17 \%$ \\
\hline & & $\mathrm{TF}$ & 4 & $10 \%$ \\
\hline & & BA & 0 & $0 \%$ \\
\hline
\end{tabular}

strategies. It is noticeable that our trading strategy with our dynamic threshold (DT-TS) gained the highest profit among all thresholds and all trading strategies. Our trading strategy with the fixed thresholds performed well and was more profitable than other trading strategies, except for the 


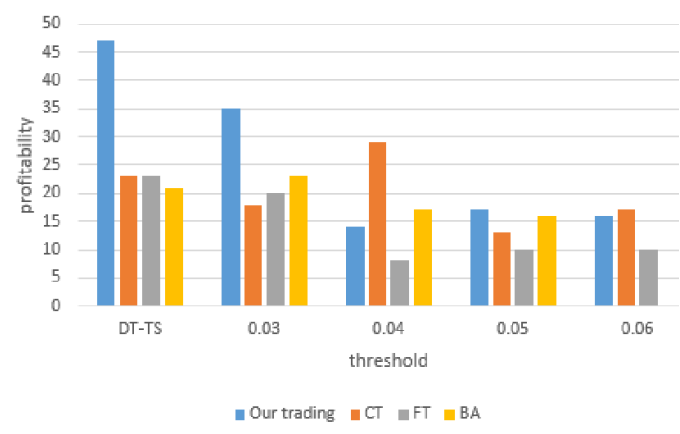

Figure 6. Profitability Comparision between Trading strategies in the Testing set

0.04 fixed threshold where the CT trading strategy was more profitable with $29 \%$ profits (an outlier). Using the 0.04 fixed threshold with our trading strategy (FT-TS), 3 out of the 10 detected DC events triggered the TF trading actions, and 7 DC events on the other hand triggered the CT trading action. Three sequential events with the CT trading actions (all regarding the EU referendum and Brexit) were not actioned upon as there was no sufficient cash for buying or shares for selling. After each one of them (the CT trading actions) and on the next day, prices were still rising or falling depending on the event but with nothing special happening the previous day, hence this triggered a TF trading action to take place, which in turns prevented the next CT trading action (belonging to the next DC event) from being satisfied.

In general, we do not want to miss a CT trading action as we are certain something has happened the previous day, and so it is an opportunity to trade. However if in the same trend, a CT trading is activated but not satisfied (no sufficient cash or shares), and is followed by a single TF trading action (the TF trading action is satisfied). Then another trend starts and a CT trading action is activated, it (the CT trading action) will also not be applied. In another words, in the same trend a not satisfied CT trading action, followed by a single TF that is satisfied, then if followed by another CT trading action (belongs to the next trend), it will also not be satisfied as the amount of money or shares is already consumed.

\section{Conclusions}

In this paper, we introduced a new trading strategy based on the Directional Change (DC) event approach with a daily dynamic calculated threshold, named the Dynamic Threshold Trading Strategy (DT-TS). The DC approach is an approach for summarizing market price movements. An event in DC is detected if the price change between two points exceeds or is below (depending on the event either downturn or upturn) the given threshold value. We applied the DC approach with a dynamic threshold that is daily calculated based on the previous day (between previous day opening and closing prices) and overnight (between the previous day closing price and current day opening price) price transitions to replace the fixed given one. A DT-TS trading rule is activated with every price increase/decrease during an upward/downward OS event. The trading decision to be taken depends on the previous day and overnight price transitions. An experiment was conducted to evaluate the DT-TS trading rules using different fixed thresholds $(0.03$, $0.04,0.05$, and 0.06) as well as different trading strategies (CT, TF, and BA). The DT-TS outperformed (gained more profits) both the fixed thresholds (FT-TS) and the investigated trading strategies.

In the future we want to apply the DT-TS on other time-series streams with different data flow levels (different frequencies, daily frequency as an example) to further investigate its effectiveness and discover whether it performs equally with data streams of different frequencies or not. In addition, we want to apply the DT-TS to other markets that are continuously streaming prices and do not close overnight (as the foreign exchange markets) and find out how would the DT-TS perform.

\section{Acknowledgments}

We acknowledge support from grant number ES/L011859/1, from The Business and Local Government Data Research Centre, funded by the Economic and Social Research Council to provide researchers and analysts with secure data services.

\section{References}

[1] J. Glattfelder, A. Dupuis, and R. Olsen, "Patterns in high-frequency fx data: discovery of 12 empirical scaling laws," Quantitative Finance, vol. 11, no. 4, pp. 599-614, 2011.

[2] D. M. Guillaume, M. M. Dacorogna, R. R. Davé, U. A. Müller, R. B. Olsen, and O. V. Pictet, "From the bird's eye to the microscope: A survey of new stylized facts of the intra-daily foreign exchange markets," Finance and stochastics, vol. 1, no. 2, pp. 95-129, 1997.

[3] E. Tsang, "Directional changes, definitions," Working Paper WP05010 Centre for Computational Finance and Economic Agents (CCFEA), University of Essex Revised 1, Tech. Rep., 2010.

[4] K. Kim, Electronic and algorithmic trading technology: the complete guide. Academic Press, 2010.

[5] M. Aloud, "Directional-change event trading strategy: Profitmaximizing learning strategy," in COGNITIVE 2015, The Seventh International Conference on Advanced Cognitive Technologies and Applications. IARIA, 2015, pp. 123-129.

[6] J. Gypteau, F. E. Otero, and M. Kampouridis, "Generating directional change based trading strategies with genetic programming," in European Conference on the Applications of Evolutionary Computation. Springer, 2015, pp. 267-278.

[7] M. Aloud, E. Tsang, R. Olsen, and A. Dupuis, "A directional-change event approach for studying financial time series," Economics: The Open-Access, Open-Assessment E-Journal, vol. 6, no. 2012-36, 2012. [Online]. Available: http://dx.doi.org/10.5018/economics-ejournal.ja. 2012-36

[8] T. Bisig, A. Dupuis, V. Impagliazzo, and R. Olsen, "The scale of market quakes," Quantitative Finance, vol. 12, no. 4, pp. 501-508, 2012.

[9] M. Aloud, M. Fasli, E. Tsang, A. Dupuis, and R. Olsen, "Stylized facts of trading activity in the high frequency fx market: An empirical study," Journal of Finance and Investment Analysis, vol. 2, no. 4, pp. 145-183, 2013. 
[10] A. Bakhach, E. P. Tsang, and W. L. Ng, "Forecasting directional changes in financial markets," Working Paper WP075-15 Centre for Computational Finance and Economic Agents (CCFEA), University of Essex, Tech. Rep., 2015.

[11] E. Tsang, R. Tao, and S. Ma, "Profiling financial market dynamics under directional changes," Working Paper WP074-15, Centre for Computational Finance and Economic Agents (CCFEA), University of Essex, Tech. Rep., 2015.

[12] M. Aloud and M. Fasli, "Exploring trading strategies and their effects in the foreign exchange market," Computational Intelligence, 2016. [Online]. Available: http://dx.doi.org/10.1111/coin.12085

[13] A. Bakhach, E. Tsang, W. L. Ng, and V. L. R. Chinthalapati, "Backlash agent: A trading strategy based on directional change," in 2016 IEEE Symposium Series on Computational Intelligence (SSCI), Dec 2016, pp. 1-9.

[14] Y. Shynkevich, T. M. McGinnity, S. Coleman, Y. Li, and A. Belatreche, "Forecasting stock price directional movements using technical indicators: investigating window size effects on one-step-ahead forecasting," in 2014 IEEE Conference on Computational Intelligence for Financial Engineering \& Economics (CIFEr). IEEE, 2014, pp. 341-348.

[15] M. E. Aloud, "Profitability of directional change based trading strategies: The case of saudi stock market," International Journal of Economics and Financial Issues, vol. 6, no. 1, 2016.

[16] E. P. Tsang, R. Tao, A. Serguieva, and S. Ma, "Profiling highfrequency equity price movements in directional changes," Quantitative Finance, pp. 1-9, 2016.

[17] M. Kampouridis and F. E. Otero, "Evolving trading strategies using directional changes," Expert Systems with Applications, vol. 73, pp. 145-160, 2017.

[18] A. Bakhach, E. P. K. Tsang, and H. Jalalian, "Forecasting directional changes in the fx markets," in 2016 IEEE Symposium Series on Computational Intelligence (SSCI), Dec 2016, pp. 1-8.

[19] S. Fong and J. Tai, "The application of trend following strategies in stock market trading," in INC, IMS and IDC, 2009. NCM'09. Fifth International Joint Conference on. IEEE, 2009, pp. 1971-1976.

[20] ThomsonReuters. Thomson one.com. [Accessed 29-2-2016]. [Online]. Available: http://www.thomsonone.com/

[21] J. R. Quinlan, C4. 5: programs for machine learning. Elsevier, 2014.

[22] I. H. Witten, E. Frank, M. A. Hall, and C. J. Pal, Data Mining: Practical machine learning tools and techniques. Morgan Kaufmann, 2016. 